\title{
Average profiles of the solar wind and outer radiation belt during the extreme flux enhancement of relativistic electrons at geosynchronous orbit
}

\author{
R. Kataoka ${ }^{1}$ and Y. Miyoshi ${ }^{2}$ \\ ${ }^{1}$ RIKEN (The Institute of Physics and Chemical Research) 2-1, Hirosawa, Wako, 351-0198, Japan \\ ${ }^{2}$ Solar-Terrestrial Environment Laboratory, Nagoya University, Furo-cho, Nagoya, Aichi, 464-8601, Japan
}

Received: 17 April 2008 - Revised: 17 May 2008 - Accepted: 21 May 2008 - Published: 11 June 2008

\begin{abstract}
We report average profiles of the solar wind and outer radiation belt during the extreme flux enhancement of relativistic electrons at geosynchronous orbit (GEO). It is found that seven of top ten extreme events at GEO during solar cycle 23 are associated with the magnetosphere inflation during the storm recovery phase as caused by the large-scale solar wind structure of very low dynamic pressure $(<1.0 \mathrm{nPa})$ during rapid speed decrease from very high $(>650 \mathrm{~km} / \mathrm{s})$ to typical $(400-500 \mathrm{~km} / \mathrm{s})$ in a few days. For the seven events, the solar wind parameters, geomagnetic activity indices, and relativistic electron flux and geomagnetic field at GEO are superposed at the local noon period of GOES satellites to investigate the physical cause. The average profiles support the "double inflation" mechanism that the rarefaction of the solar wind and subsequent magnetosphere inflation are one of the best conditions to produce the extreme flux enhancement at GEO because of the excellent magnetic confinement of relativistic electrons by reducing the drift loss of trapped electrons at dayside magnetopause.
\end{abstract}

Keywords. Magnetospheric physics (Magnetotail; Storms and substorms) - Space plasma physics (Nonlinear phenomena)

\section{Introduction}

The Van Allen radiation belts are composed of ions and electrons ranging from hundreds of $\mathrm{keV}$ to tens of $\mathrm{MeV}$. The outer radiation belt usually exists at $L=3.5-7.0$ where GPS satellites, metrological satellites, broadcasting and communication satellites are operating. Deep dielectric charging can occur when high fluxes of relativistic electrons penetrate the spacecraft shielding and imbed themselves in spacecraft

Correspondence to: R. Kataoka

(ryuho@ riken.jp) dielectrics such as coaxial cables and circuit boards. Discharges and subsequent damages tend to occur after long periods of the high fluxes, and these effects have been demonstrated in the laboratory (Baker et al., 1987, 1998). Understanding the physical cause of extremely high flux of outer belt electrons is therefore important for the space weather forecast of the potentially problematic satellite environment. Two major mechanisms have been suggested for the fundamental acceleration process of outer belt electrons (e.g. Friedel et al., 2002): Pc5-type ULF waves can work as a driver for the adiabatic radial diffusion (e.g. Elikington et al., 1999), while whistler-mode chorus VLF waves are another candidate to cause the non-adiabatic acceleration via waveparticle interactions (e.g. Miyoshi et al., 2003).

It has been well known that the outer belt electrons tend to increase associated with high-speed solar wind (e.g. Paulikas and Blake, 1979). Recently, Miyoshi and Kataoka (2008a) showed that the flux enhancement depends not only on the solar wind speed but also on the interplanetary magnetic field (IMF) $B_{z}$ offset as determined by the sector polarity of the IMF and seasons, via so-called "spring toward fall away" (STFA) rule of Russell and McPherron (1973). Miyoshi et al. (2007) showed that the STFA rule controls the nonadiabatic acceleration for outer belt electrons by the whistlermode chorus wave-particle interactions during HILDCAAs (High Intensity Long Duration Continuous Substorm Activities) (Tsurutani and Gonzalez, 1987). Also, Miyoshi and Kataoka (2005) showed that the high-speed solar wind associated with corotating interaction regions (CIRs) is more effective for the flux enhancement during the storm recovery phase on average than the high-speed solar wind associated with coronal mass ejections (CMEs). Based on the findings of the solar wind driver dependence, Kataoka and Miyoshi (2006) developed an algorithm of a probabilistic forecast of the NOAA alert level of relativistic electrons at GEO, and the algorithm was further extended to general

Published by Copernicus Publications on behalf of the European Geosciences Union. 
Table 1. Event list of top 10 extreme flux enhancements determined from GOES-8 and 12 observations during solar cycle 23 . From left to right, columns show the maximum flux of $>2.0 \mathrm{MeV}$ electrons at $\mathrm{GEO}$ ( $\mathrm{pfu}=/ \mathrm{cm}^{2} \mathrm{~s} \mathrm{str}$ ), date of the event, minimum daily variation of $\mathrm{H}$ component at GEO in the VDH-coordinate system from $t=-2.0$ to 0.0 days, minimum solar wind dynamic pressure, maximum solar wind speed, solar wind driver, the STFA relationship, and HILDCAAs occurrence.

\begin{tabular}{|c|c|c|c|c|c|c|c|}
\hline $\begin{array}{c}\max \text { flux } \\
\log 10(p f u)\end{array}$ & $\begin{array}{c}\text { date } \\
\text { yyyymmdd }\end{array}$ & $\begin{array}{c}\min d H \\
\mathrm{nT}\end{array}$ & $\begin{array}{c}\min P d \\
\mathrm{nPa}\end{array}$ & $\begin{array}{c}\max V \\
\mathrm{~km} / \mathrm{s}\end{array}$ & $\begin{array}{l}\text { solra wind } \\
\text { driver }\end{array}$ & $\begin{array}{l}\text { STFA } \\
\text { rule }\end{array}$ & HILDCAAs \\
\hline 5.223 & 20040729 & 19.7 & 0.27 & 1027 & $\mathrm{CME}+\mathrm{CHS}$ & summer & no \\
\hline 4.936 & 20050518 & 24.6 & 0.22 & 959 & CME & spring-to & yes \\
\hline 4.876 & 20050919 & 17.5 & 0.32 & 862 & not clear & fall-away & no \\
\hline 4.719 & 20060417 & 22.2 & 0.56 & 676 & CHS & spring-to & possible \\
\hline 4.691 & 20050809 & 27.3 & 0.46 & 714 & $\mathrm{CME}+\mathrm{CHS}$ & summer & no \\
\hline 4.689 & 20040218 & 20.3 & 0.08 & 735 & CHS & winter & possible \\
\hline 4.674 & 20041111 & 64.3 & 1.21 & 809 & CME & fall-away & no \\
\hline 4.660 & 20061215 & 86.6 & 0.77 & 896 & CME & winter & no \\
\hline 4.629 & 20030920 & 45.7 & 0.77 & 812 & CHS & fall-away & yes \\
\hline 4.590 & 20050905 & 17.9 & 0.68 & 743 & not clear & fall-away & no \\
\hline
\end{tabular}

stream interface events based on the STFA rule (Miyoshi and Kataoka, 2008b) to operate the probabilistic forecast (http://hbksw1.stelab.nagoya-u.ac.jp/).

Usual flux enhancement can be predicted even in real-time operation as shown above. However, the extreme flux enhancement of relativistic electrons at GEO is still hard to predict and the generation mechanism remains unknown. More recently, Kataoka and Miyoshi (2008) showed evidence that the largest increase of outer belt electrons at GEO during solar cycle 23 is associated with the magnetosphere inflation due to very low dynamic pressure of the solar wind. The very low dynamic pressure at the trailing part of a CME is produced by the speed difference between the very fast CME and following a coronal hole stream (CHS). The purpose of this paper is to show average profiles of the solar wind and outer radiation belt during the extreme flux enhancement of relativistic electrons at GEO as listed by Kataoka and Miyoshi (2008) to investigate the mechanism of the extreme events in more detail.

\section{Results and discussions}

Table 1 shows the event list of the Kataoka and Miyoshi (2008) with some additional information. In order to create the top 10 event list, daily maximum flux (1st column) was calculated for $>2.0 \mathrm{MeV}$ electron flux measured at GOES 8 and 12 during solar cycle 23 (1996-2006). Kataoka and Miyoshi (2008) discussed a favorable condition of extreme events as coronal hole stream, storm recovery phase, and very low dynamic pressure. The last two columns show additional information on the solar wind driver: The combination of CME and CHS (July 2004, August 2005), CHS (February 2004, September 2003, April 2006), and CME (November 2004, May 2005, December 2006) can be possible solar wind structures to produce the effective solar wind pro- file for the extreme events at GEO. It is interesting to note that all of the extreme events, except for those in summer and winter, follow the STFA rule. In order to find a meaningful average profiles, the extreme events on 20 September 2003, 11 November 2004, and 15 December 2006 are removed from the following superposed epoch analysis because the geomagnetic field daily variation (3rd column) and dynamic pressure (4th column) are significantly larger than other events, implying some different mechanisms. The data source is summarized as follows: The OMNI2 hourly solar wind data is provided from NASA/SPDF (http://spdf.gsfc. nasa.gov/), GOES electrons and magnetic field and POES particle data are provided from NOAA/NGDC (http://www. ngdc.noaa.gov/). The $D_{s t}$ index is provided from Kyoto University (http://swdcwww.kugi.kyoto-u.ac.jp/). The existence of CME is confirmed using LASCO CME catalog (http://cdaw.gsfc.nasa.gov/cme_list/). The particle data from GOES-12 and NOAA/POES-15 are used for the following analysis since all of the extreme events occurred after 2003.

Figure 1 shows the average profiles of solar wind parameters and geomagnetic activities superposed at 17:00 UT of the day of maximum flux at GEO. Note that GOES-12 is roughly located at the local noon at 17:00 UT where the electron flux usually becomes largest because the magnetic field is highly compressed at noon. It is found from Fig. 1 that the maximum flux occurs when the dynamic pressure has very low value of $<1.0 \mathrm{nPa}$ for two days during the storm recovery phase, as indicated by two vertical dahsed lines. The estimated magnetopause distance (Shue et al., 1998) of $>12 R_{E}$ is also very large. The typical values of dynamic pressure and magnetopause distance are $2.0-3.0 \mathrm{nPa}$ and $10-11 R_{E}$, respectively, as also indicated at the start and end of Fig. 1. The maximum solar wind speed of $>650 \mathrm{~km} / \mathrm{s}$ is also very fast, as can be seen from Table 1 and Fig. 1, and the very fast speed and subsequent rapid speed decrease is the essential 

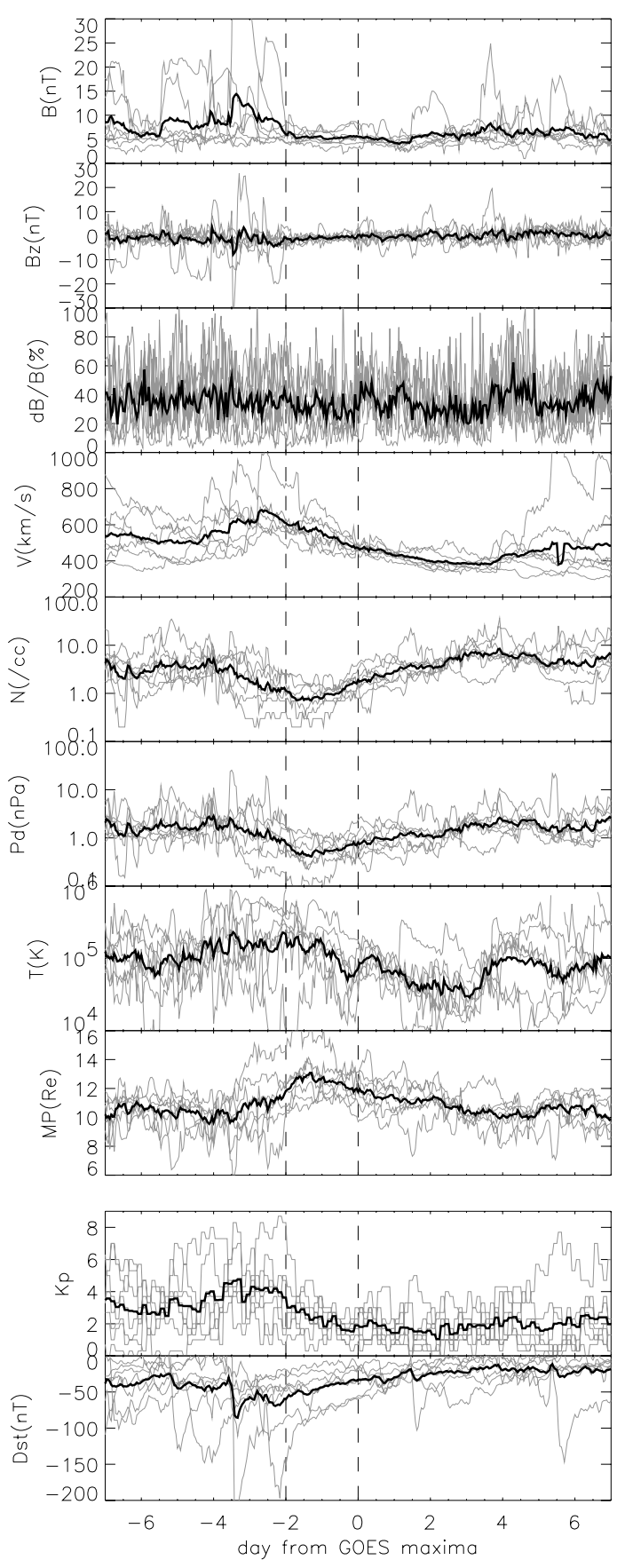

Fig. 1. Average profiles of solar wind parameters and geomagnetic activities during extreme events superposed at 17:00 UT of the day of maximum flux enhancement: From top to bottom, shown are interplanetary magnetic field strength $B$, the southward component $B_{z}$ in the GSM coordinate system, magnetic field fluctuation level $d B / B$, solar wind speed $V$, proton number density $N$, dynamic pressure $P d$, proton temperature $T$, subsolar magnetopause distance $M P, K_{p}$ index, and $D_{s t}$ index. Light gray curves show the profiles of each event. Vertical dashed lines indicate the period when the dynamic pressure has very low value of $<1.0 \mathrm{nPa}$.

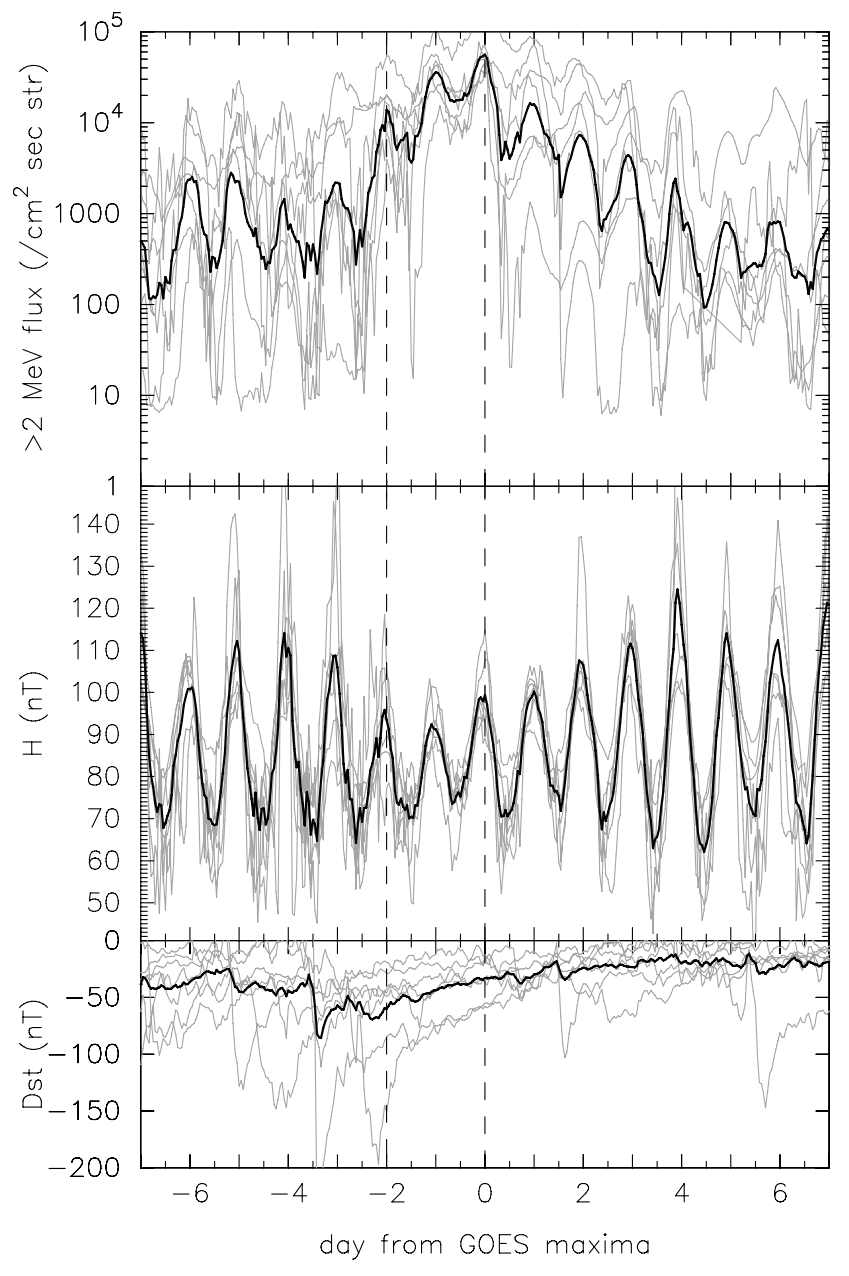

Fig. 2. Average profiles of GOES-12 $>2.0 \mathrm{MeV}$ electron flux and magnetic field at GEO during extreme events superposed at 17 UT of the day of maximum flux enhancement: (top) $>2.0 \mathrm{MeV}$ electron flux, (middle) magnetic filed $\mathrm{H}$ component in the VDH-coordinate system, (bottom) the $D_{s t}$ index. In order to avoid any contaminations from the solar protons, the electron data are not used when the flux of the $9-15 \mathrm{MeV}$ proton sensor is larger than $10 / \mathrm{cm}^{2} \mathrm{sec}$ str. Light gray curves show the profiles of each event. Vertical dashed lines indicate the period when the dynamic pressure has very low value of $<1.0 \mathrm{nPa}$ as shown in Fig. 1 .

cause of the solar wind rarefaction structure. It is important to note here that a lot of much faster solar wind structures such as very fast CMEs cannot always produce the large enhancement at GEO (e.g. Miyoshi and Kataoka, 2005), and it is true that a very fast solar wind is not a sufficient condition for producing large enhancement events at GEO (Miyoshi and Kataoka, 2008a).

Figure 2 shows the average profiles of $>2.0 \mathrm{MeV}$ electron flux and magnetic field $\mathrm{H}$ component measured by GOES-12 superposed at 17:00 UT of the day of maximum flux, where the $\mathrm{H}$ component is positive in direction of anti-parallel to the dipole axis in the VDH-coordinate system. The extreme 


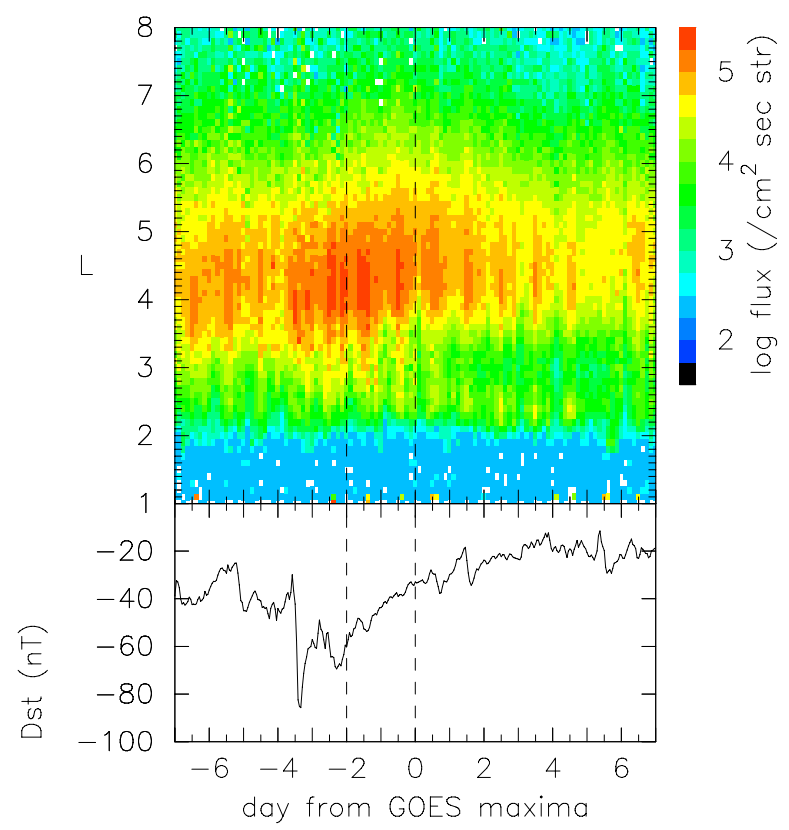

Fig. 3. Average $L$-t diagram of NOAA-15 300-2500 keV electron flux during extreme events superposed at 17:00 UT of the day of maximum flux enhancement. The $L$-value is Mcllwain's $L$ derived from the IGRF. The $D_{s t}$ index is also shown in the bottom panel. In order to avoid any contamination from the energetic protons, the electron data are not used when the electron flux of the NOAA/MEPED 300-2500 keV sensor is less than ten times of the ion flux of the NOAA/MEPED $240-800 \mathrm{keV}$ sensor.

enhancement begins two days before the maximum flux during the storm recovery phase, when the daily variation of the total magnetic field is getting smaller than usual due to the very low solar wind dynamic pressure (Fig. 1). This means that the magnetic field configuration is more dipole-like than usual when the extreme flux enhancement takes place at GEO. As a reference, average amplitude of the minimum $\mathrm{H}$ component daily variation during the recovery phase of intense CIR storms of Miyoshi and Kataoka (2005) is $52.7 \mathrm{nT}$, while that of Fig. 2 is only $21.3 \mathrm{nT}$. It is also worthwhile to note here that the flux significantly decrease after the flux maximum as following the magnetopause distance retreating from the inflation state. Ukhorskiy et al. (2006) showed from their test-particle simulation that the change of electron trajectory causes a significant loss of the relativistic electrons at magnetopause. They showed that a rapid enhancement of solar wind dynamic pressure moves the magnetopause earthward, causing the immediate electron loss from the outermost trapped region. Considering this simulation, it is expected that the inflation of the magnetosphere due to small dynamic pressure reduces such loss process at the magnetopause. Since the outer belt electron flux is determined by the delicate balance between source and loss processes (Reeves et al., 2003), reducing the loss process at $t=-2.0$ to 0.0 can naturally induce the flux enhancement.
Figure 3 shows the average profiles of 300-2500 keV electron flux measured by NOAA/POES-15 MEPED (Evans and Greer, 2000) superposed at 17:00 UT of the day of maximum flux. Note that the NOAA/POES-15 orbits 07:30-19:30 local time meridian. It is found that the outer belt shows outward expansion of the outer boundary two days before flux maximum and then shrinks after the flux maximum. The outward and inward motion of the outer belt is consistent with the flux variation at GEO as shown in Fig. 2. The flux amplitude around the peak of the outer belt $(L=4.0-4.5)$ is comparable to that of intense CIR storms (see Miyoshi and Kataoka, 2005), suggesting that the acceleration source itself at the heart of the outer belt is not extremely strong. In fact, average $K_{p}$ index of extreme events during the recovery phase (Fig. 1) is comparable or smaller than that of intense CIR storms (Miyoshi and Kataoka, 2005), implying comparable or smaller energy source for accelerating relativistic electrons. It is also found that the extreme events are not always associated with HILDCAAs. In Table 1, the association with HILDCAAs is marked as "yes" if HILDCAAs occur during the day of maximum flux, and is marked as "possible" if HILDCAAs occur 1-2 days before the day of maximum flux. The weak association with HILDCAAs is consistent with the fact that the strength of Alfvénic fluctuation of the solar wind during the extreme events is not strong on average as shown in the third panels of Fig. 1.

In summary, all of the observations shown above are consistent with the "double inflation" mechanism (Kataoka and Miyoshi, 2008) that the solar wind rarefaction and subsequent magnetosphere inflation essentially cause the extreme flux enhancement at GEO via the excellent magnetic confinement of relativistic electrons by reducing the drift loss of trapped electrons at magnetopause. The fundamental acceleration mechanism of relativistic electrons during the extreme events is, however, still not clear from the results of this paper, and further research and new observations of the phase space density profiles and plasma wave activities are definitely needed to clarify the acceleration process. Direct evidence of the drift loss process that relativistic electrons escape from the magnetopause is also necessary to understand the mechanism because there are some other possible explanations for the loss process. For example, Green et al. (2004) concluded that the electron decreases are likely due to enhanced precipitation into the atmosphere by EMIC waves. The planned satellite missions for the inner magnetosphere during the solar maximum of solar cycle 24 such as ORBITALS, RBSP within the International Living With a Star (ILWS) program, and Japanese ERG project (Shiokawa et al., 2006) will provide the essential data for elucidating the fundamental acceleration and loss mechanisms of relativistic electrons of the radiation belts. 
Acknowledgements. The OMNI-2 data are provided from NASA/NSSDC. The GOES and POES satellite data are provided from NOAA/NGDC. The $D_{s t}$ index and the provisional and quick-link AE index are provided from Kyoto University. The work by R. Kataoka was supported by a research fellowship of Special Postdoctoral Research Program at RIKEN. This work was supported by the Grant-in-Aid for Creative Scientific Research "The Basic Study of Space Weather Prediction" (17GS0208, Head Investigator: K. Shibata) from the Ministry of Education, Science, Sports, Technology, and Culture of Japan, and the Housou Bunka Foundation, Japan.

Topical Editor I. A. Daglis thanks one anonymous referee for her/his help in evaluating this paper.

\section{References}

Baker, D. N., Belian, R. D., Higbie, P. R., Klebesadel, R. W., and Blake, J. B.: Deep dielectric charging effects due to high energy electrons in the Earth's outer magnetosphere, J. Electrost., 20, 3-19, 1987.

Baker, D. N., Allen, J. H., Kanekal, S. G., and Reeves, G. D.: Disturbed space environment may have been related to pager satellite failure, EOS, 79, 477, 1998.

Elkington, S. R., Hudson, M. K., and Chan, A. A.: Acceleration of relativistic electrons via drift-resonant interaction with toroidalmode Pc5 ULF oscillations, Geophys. Res. Lett., 26, 3273-3276, 1999.

Evans, D. S. and Greer, M. S.: Polar orbiting environmental satellite space environment monitor:2. Instrument description and archive data documentation, NOAA Tech. Memo. OAR SEC-93, Natl. Oceanic and Atmos. Admin., Boulder, Colorado, 2000.

Friedel, R. H. W., Reeves, G. D., and Obara, T.: Relativistic electron dynamics in the inner magnetosphere- A review, J. Atmos. SolarTerr. Phys., 64, 265-282, 2002.

Green, J. C., Onsager, T. G., O’Brien, T. P., and Baker, D. N.: Testing loss mechanism capable of rapidly depleting relativistic electron flux in the Earth's outer radiation belt, J. Geophys. Res., 109, A12211, doi:10.1029/2004JA010579, 2004.

Kataoka, R. and Miyoshi, Y.: Flux enhancement of radiation belt electrons during geomagnetic storms driven by coronal mass ejections and corotating interaction regions, Space Weather, 4, S09004, doi:10.1029/2005SW000211, 2006.

Kataoka, R. and Miyoshi, Y.: Magnetosphere inflation during the recovery phase of geomagnetic storms as an excellent magnetic confinement of killer electrons, Geophys. Res. Lett., 35, L06S09, doi:10.1029/2007GL031842, 2008.
Miyoshi, Y., Morioka, A., Obara, T., Misawa, H., Nagai, T., and Kasahara, Y.: Rebuilding process of the outer radiation belt during the November 3, 1993, magnetic storm - NOAA and EXOS-D observations, J. Geophys. Res., 108(A1), 1004, doi:10.1029/2001JA007542, 2003.

Miyoshi, Y., Morioka, A., Kataoka, R., Kasahara, Y., and Mukai, T.: Evolution of the outer radiation belt during the November 1993 storms driven by corotating interaction regions, J. Geophys. Res., 112, A05210, doi:10.1029/2006JA012148, 2007.

Miyoshi, Y. and Kataoka, R.: Ring current ions and radiation belt electrons during geomagnetic storms driven by coronal mass ejections and corotating interaction regions, Geophys. Res. Lett., 32, L21105, doi:10.1029/2005GL024590, 2005.

Miyoshi, Y. and Kataoka, R.: Flux enhancement of the outer radiation belt electrons associated with stream interaction regions, J. Geophys. Res., 113, A03S09, doi:10.1029/2007JA012506, 2008a.

Miyoshi, Y. and Kataoka, R.: Probabilistic space weather forecast of the relativistic electron flux enhancement at geosynchronous orbit, J. Atmos. Sol. Terr. Phys., 70, 475-481, 2008b.

Paulikas, G. A. and Blake, J. B.: Effects of the solar wind on magnetospheric dynamics: Energetic electrons at the synchronous orbit, in Quantitative modeling of magnetospheric processes, edited by W. P. Olson, AGU monograph 21, American Geophysical Union, Washington D.C., 1979.

Reeves, G. D., McAdams, K. L., Friedel, R. H., and O'Brien, T. P.: Acceleration and loss of relativistic electrons during geomagnetic storms, Geophys. Res. Lett., 30(10), 1529, doi:10.1029/2002GL016513, 2003.

Russell, C. T. and McPherron, R. L.: Semiannual variation of geomagnetic activity, J. Geophys. Res., 78, 92-108, 1973.

Shiokawa, K., Seki, K., Miyoshi, Y., et al.: ERG - A small-satellite mission to investigate the dynamics of the inner magnetosphere, Adv. Space Res., 38(8), 1861-1869, 2006.

Shue, J.-H., Song, P., Russell, C. T., Steinberg, J. T., Chao, J. K., Zastenker, G., Vaisberg, O. L., Kokubun, S., Singer, H. J., Detman, T. R., and Kawano, H.: Magnetopause location under extreme solar wind conditions, J. Geophys. Res., 103(A8), 17 69117 700, doi:10.1029/98JA01103, 1998.

Tsurutani, B. T. and Gonzalez, W. D.: The cause of high-intensity long-duration continuous AE activity (HILDCAAs): Interplanetary Alfven wave trains, Planet. Space Sci., 35, 305-412, 1987.

Ukhorskiy, A., Y., Anderson, B. J., Brandt, P. C., and Tsyganenko, N. A.: Storm time evolution of the outer radiation belt: Transport and losses, J. Geophys. Res., 111, A11S03, doi:10.1029/2006JA011690, 2006. 